\title{
Leistung und Perspektiven der modernen Pathologie in der Tumordiagnostik
}

\author{
R. Parwaresch P. Rudolph \\ Institut für Hämatopathologie und Lymphknotenregister des Universitätsklinikum Kiel
}

\section{Einleitung}

In der Tumordiagnostik bedient sich die moderne Medizin immer besserer und komplexerer Technologien. Die großen Fortschritte in den bildgebenden Verfahren und die Entwicklung der chemischen Tumormarker werfen die Frage nach der aktuellen und zukünftigen Rolle des Pathologen besonders in der modernen Tumordiagnostik auf.

In dieser Abhandlung soll der Aufgabenkreis des Pathologen in der Tumordiagnostik und im Hinblick auf das «Krebsmanagement» dargestellt und sein Tätigkeitsradius vor dem Hintergrund der modernen Fortschritte in der biomedizinischen Grundlagenforschung und der klinischen Medizin an konkreten Beispielen gezeigt werden. Sowohl in seiner autoptischen als auch in der bioptischen Tätigkeit bilden die Beiträge des Pathologen das Fundament der Krebsbehandlung.

Die bioptische Histopathologie macht den Löwenanteil der Tätigkeit eines Pathologen und damit auch seinen eigentlichen Beitrag zur klinischen Onkologie aus. Die histopathologische Diagnose ist somit das Fundament, auf dem das Konzept der Krebsbehandlung ruht. Hier trägt der Pathologe die Hauptlast der Verantwortung. Seine Maxime ist und bleibt, die richtige und genaue Diagnose zu stellen; es gilt für heute wie für die Zukunft die Leitlinie: Mit der Diagnose steht und fällt eine jede Behandlungsstrategie. Die Bedeutung einer richtigen und in ihren Substrukturen genau gestellten Diagnose wird in Zukunft noch mehr als bisher ins Gewicht fallen, weil immer mehr therapeutische Optionen verfügbar werden. Heute muss ein Tumor in seiner geweblichen Zusammensetzung, Organzugehörigkeit und seiner biologischen Dignität näher charakterisiert werden. Dabei ist die Diagnostik Schritt für Schritt voran zu treiben und zu verfeinern. Darüber hinaus bedarf die Tumordiagnose weiterer Informationen über Größe, Befall der ersten Lymphknotenstationen («Sentinel»Lymphknoten), Atypiegrad der Tumorzellkerne («Nuclear
Grading»), histopathologischer Malignitätsgrad (z.B. BloomRichardson-Grading beim Mammakarzinom), Hormonrezeptorausstattung, Ansprechbarkeit für bestimmte Behandlungsstrategien wie eine Chemotherapie mit Inhibitoren der Topoisomerase-I $\alpha$ - oder -II $\alpha$ [26] oder monoklonalen Antikörpern wie Herzeptin gegen das Proteinprodukt des Her-2/ neu-Gens oder Rituximab [1,2].

\section{Die «Werkzeuge» des Pathologen}

Karzinome treten in weit gefächerten Varianten auf, die nur zum Teil auf ihr Muttergewebe schließen lassen. Das Ursprungsgewebe kann bei Metastasen entsprechend auch nur in einem kleinen Teil sicher bestimmt werden. Dies gilt z.B. für bestimmte Schilddrüsenkarzinome, die auf Grund des typischen papillären Aufbaues und des Amyloid- oder Thyreoglobulingehaltes identifiziert werden. Ähnlich können häufig die Tumoren der endokrinen oder neuroendokrinen Epithelien, wie das Paragangliom oder Merkelzelltumor, durch ihren Gehalt an Neuropeptiden oder einen bestimmten Zytokeratintyp in ihrer Herkunft definiert werden.

Bei Keimzelltumoren kann die plazentaspezifische alkalische Phosphatase, sicherer aber das «Cancer-Testis Antigen» p24/ 28 mit dem monoklonalen Antikörper Ki-A10 diagnostisch oder differentialdiagnostisch herangezogen werden [3].

Maligne Melanome ohne Melanin (amelanotische Leukometastasen) können große diagnostische Schwierigkeiten bereiten, speziell wenn sie primär extrakutan vorkommen und eine kleinzellige lymphomähnliche Morphologie annehmen. Gute Hilfe leisten hier die Antigene S-100 und HMB-45 und die Abwesenheit von Zytokeratin, CD45 und Ki-M1p.

Maligne Mesotheliome können immer noch nicht sicher von Adenokarzinomen getrennt werden. Allerdings erweist sich in einem Teil der Fälle der Nachweis von Thrombomodulin und

\begin{tabular}{ll}
\hline KARGER & @ 2002 S. Karger GmbH, Freiburg \\
Fax +49 7614520714 & Accessible online at: \\
$\begin{array}{l}\text { E-mail Information@Karger.de } \\
\text { www.karger.com }\end{array}$ & www.karger.com/journals/onk
\end{tabular}

Prof. Dr. Dr. h.c. R. Parwaresch

Institut für Hämatopathologie und Lymphknotenregister

des Universitätsklinikum Kiel

Niemannsweg 11

D-24105 Kiel (Deutschland) 


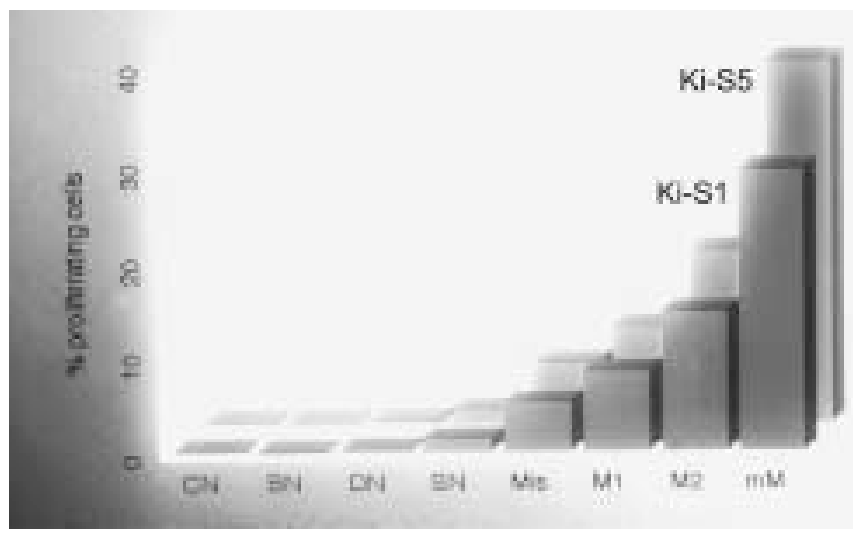

Abb. 1. Proliferationsaktivität von verschiedenen naevozellulären Tumoren gemessen mit den monoklonalen Antikörper Ki-S5 gegen Ki-67 und Ki-S1 gegen die humane Topoisomerase IIa. $\mathrm{CN}=$ Common Naevi; $\mathrm{BN}=$ Benign Naevi; DN = Dysplastic Naevi; $\mathrm{SN}=$ Spitz Naevi; Mis = Melanoma in situ; M1 = Flaches Melanom; M2 = Noduläres Melanom; $\mathrm{mM}=$ Metastatisches Melanom [5].

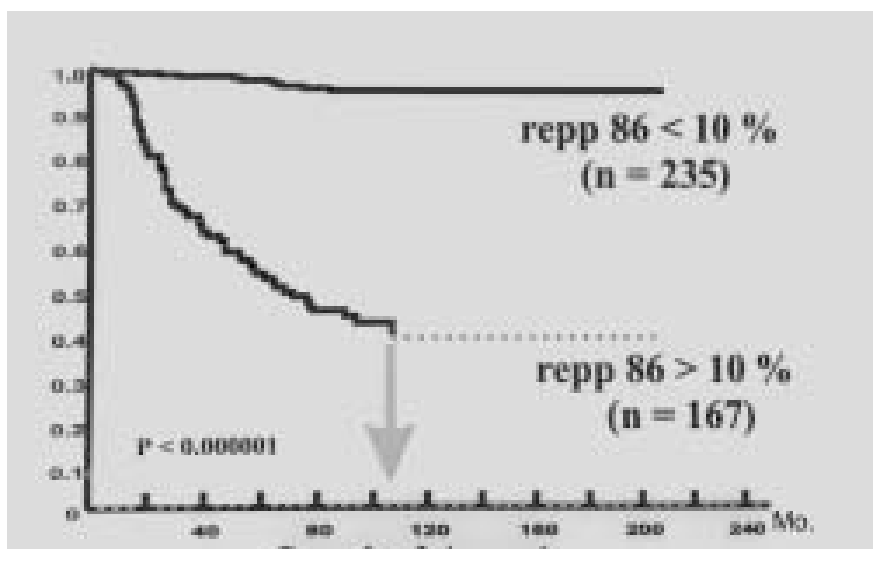

Abb. 2. Überlebenskurven von Patientinnen mit nodal negativen Mammakarzinomen (T1 \& 2 N0 M0) nach einheitlicher Lumpektomie und örtlicher Bestrahlung bei einer «Follow-up» Zeit von über 200 Monaten. Die prozentuale Expression von Repp86 in den Karzinomzellen ist ein verlässliches prognostisches Kriterium [16].

Calretinin als hilfreich. Ausbreitung und anamnestische Informationen über eine Asbest-Exposition können zusätzliche Anhaltspunkte liefern.

Sarkome, besonders die Weichteilsarkome, lassen sich nur teilweise auf Grund eines immunphänotypischen Merkmals sicher diagnostizieren. Das Vorkommen von Desmin in hohen Konzentrationen zeigt eine rhabdomyomatöse Abstammung an. Endotheliale Antigene, wie Faktor VIII, CD34 und CD31, erlauben, eine angiomatöse Differenzierung zu erfassen. Leiomyosarkome enthalten das Aktin der glatten Muskelzellen. Chondro-, Lipo-, Osteo-, Neurosarkome lassen sich auf Grund spezifischer Strukturen (Lipoblasten, Knorpel, Knochen oder Osteoid, Neurosekrete) typisieren. Viele Merkmale der genannten Sarkome können jedoch in den verschiedenen
Varianten des malignen fibrösen Histiozytoms (MFH) enthalten sein. Dies ist auch der Grund, warum das MFH von manchen Autoren als das Sarkom der pluripotenten mesenchymalen Stammzelle angesehen wird. Eigenartigerweise exprimiert das MFH nicht selten hochspezifische monozytäre Merkmale wie Ki-M1p [4].

Dem Pathologen und Neuropathologen stellen sich täglich eine Vielzahl von besonders schwierigen diagnostischen und differentialdiagnostischen Aufgaben in der Erkennung und Typisierung von malignen Neoplasien des Gehirns und der peripheren Nerven. Erschwerend kommt hierzu die Brisanz der Probengewinnung, die auf ein notwendiges Minimum begrenzt sein muss. Dabei ist es nicht nur problematisch, die Tumoren zu typisieren, sondern es treten auch zuweilen Probleme bei der Abgrenzung von normalem oder reparativem Gewebe gegenüber malignen Neoplasien auf.

Die Dermatopathologie der Hauttumoren gehört zu den schwierigsten Kapiteln der Histopathologie und bedarf entsprechender dermatopathologischer Erfahrung. Bereits die Abgrenzung einer Akanthose von einem Plattenepithelkarzinom kann erhebliche Probleme bereiten. Keratoakanthome können von einem hoch differenzierten Plattenepithelkarzinom manchmal gar nicht unterschieden werden. Ähnlich können Basalzellhyperplasien z.B. über einem dermalen Histiozytom, einer Fibrose, einer Entzündung usw. von einem Basalzellkarzinom nicht immer hinreichend sicher abgegrenzt werden. Ähnliche zum Teil fatale Unsicherheiten bestehen bei der Diagnose von Weichteilsarkomen der Haut, die wegen ihrer oberflächlichen Lokalisation rechtzeitig in sano entfernt werden könnten. Neben malignen fibrösen Histiozytomen, Leiomyosarkomen, Rhabdomyosarkomen und Angiosarkomen gehören auch desmoplastische Varianten des malignen Melanoms, maligne blaue Naevi und die desmoplastischen und pseudosarkomatösen Varianten des Plattenepithelkarzinoms in diese Reihe [4].

Melanozytäre Hautläsionen umfassen das Spektrum von flachen, oberflächlichen intraepithelialen Naevi bis hin zu nodulären malignen Melanomen. Die sachgemäße Einordnung der einzelnen Entitäten und die adäquate Würdigung ihrer biologischen Dignität erfordert den Einsatz neuerer immunhistochemischer Methoden. Hier bewährt sich die Untersuchung der Proliferationsaktivität, um den Wachstumsdruck und die Aggressivität des Tumors und damit seinen wahren Charakter $\mathrm{zu}$ enthüllen. Es konnte gezeigt werden, dass sich die benignen und die malignen Pigmenttumoren durch ihre Proliferationsaktivität (Abb. 1) unterscheiden [5].

Ein eigenes Kapitel bilden auch die lymphatischen Hautinfiltrate. Diese variieren von einfachen immunologisch bedingten entzündlichen Reaktionen bis hin zu hoch aggressiven malignen Lymphomen, deren Einschätzung neben dermatopathologischer Erfahrung auch die sachgerechte Anwendung moderner hämatologischer Methoden erfordert, die von der einfachen Rearrangementanalyse der Immungene bis hin zur Aufdeckung von genetischen Expressionsdefekten z.B. im Be- 
Tab. 1. Reaktivität der Zellzyklus-assoziierten Proteine in den Zellzyklusphasen: S, G2, M, G1 und G0-Ruhephase

\begin{tabular}{llllllll}
\hline Protein & MAK & S & G2 & M & G1 & G0 \\
\hline Ki-67 & Mib1, Ki-S5, Ki-S11 & + & + & + & + & - \\
Topoisomerase-II $\alpha$ & Ki-S1, Ki-S4 & + & + & + & + & - \\
repp86 & Ki-S2, Anti-repp86-1,2 & + & + & + & - & - \\
\hline
\end{tabular}

Das Protein repp86 wird in der G1-Phase des Zellzyklus nicht exprimiert. Übersicht siehe [25]. reich der Palette der proapoptotischen Gene wie CD95 und LyGDI reicht.

In der Einschätzung der biologischen Dignität und in der Voraussage der Prognose von malignen Tumoren finden auch zunehmend Zellzyklusproteine wie die Zykline, ZyklinkinaseInhibitoren und die Proliferationsmarker (Tab. 1) Anwendung. Damit kann die Invasivität der Behandlung bereits bei der Erstellung der primären Diagnose besonders bei Mammakarzinomen in den Stadien T1 und 2, N0, M0 festgelegt werden. Proliferationsmarker der ersten Generation, wie die Topoisomerase-II $\alpha$ und das nukleäre Protein Ki-67, die mit den monoklonalen Antikörpern Ki-S1, Mib-1, Ki-S5 und Ki-S11 meist immunhistochemisch dargestellt werden, erlauben eine gewisse Aussage über die Aggressivität der untersuchten Malignome. Bei einer Reihe von Tumoren sind solche Untersuchungen mit großem Erfolg bereits durchgeführt worden. Hierzu gehören: Mammakarzinome [6, 7], Neuroblastome [8, 9], Nierenzellkarzinome [10], Weichteilsarkome [11], gastrointestinale Stromatumoren [12], melanozytäre Tumoren [5, 13], endometriale Karzinome [14] und maligne Lymphome [15], einschließlich der Plasmazell-Myelome oder der Plasmozytome.

Eine verlässliche Korrelation zwischen der Prognose und dem Prozentsatz Ki-67-positiver Tumorzellen bestand aber nicht in allen Fällen. Diese und ähnliche Mängel haben die generelle Anwendung dieser einfachen und an sich plausiblen Methode erheblich eingeschränkt. Die ungenügende Korrelation solcher Proliferationsmarker mit der Prognose ist darin begründet, dass die Proteine wie Ki-67 oder die Topoisomerase-II $\alpha$ zwar proliferationsassoziiert sind und in ruhenden Zellen in G0 nicht vorkommen, sie werden allerdings gleichermaßen in allen Zellzyklusphasen, d.h. G1, S, G2, und M exprimiert. Während die mittlere Dauer der Zellzyklusphasen S, G2 und $\mathrm{M}$ mit jeweils 10,5 und $1 \frac{1}{2} \mathrm{~h}$ unter normalem «steady-stateequilibrium» des Zellumsatzes weitgehend konstant bleibt, variiert G1 mit einer Dauer von 0 bis mehreren Jahren erheblich [25]. Die Variabilität der G1-Phase wird durch Unterschiede im Nahrungsangebot, $\mathrm{pH}$, Sauerstoffversorgung, Zellkontakt und Konzentration von Wachstumsfaktoren bedingt. Bei langen G1-Phasen spiegelt die Positivität der Zellen für Ki-67 oder Topoisomerase-II $\alpha$ nur partiell die Progressionstendenz des Tumors wider. Hierauf beruhen auch die Mängel in der Korrelation zwischen den sogenannten Proliferationsraten und der Tumorprognose.
Tab. 2. Hämatopoetische Neoplasien, die CD45-negativ sein können.

Precursor lymphoblastische Leukämien/Lymphome

Anaplastische Lymphome (Ki1-Lymphome)

Plasmazell-Myelome

Echte maligne Histiozytose

Stammzellnahe Leukämien

Natural Killer Cell Lymphome

Proliferationsmarker der zweiten Generation haben diesen Mangel nicht, da sie nur ein eingeschränktes Spektrum der Zellzyklusphasen erfassen. Ein Beispiel hierfür ist das «restrictedly expressed proliferation-associated protein 86» (repp86). Dieses Protein interagiert mit anderen chromosomalen Segregationsproteinen und wird ausschließlich in den Zellzyklusphasen S, G2 und M gebildet. repp86 zeigt daher den erfolgreichen Ablauf der Zellzyklusabschnitte vom Restriktionspunkt G1/S bis hin zum Vollzug der Mitose an. Damit entfallen alle unvorhersehbaren Abweichungen durch die Variabilität der G1-Phase. Entsprechend besteht auch in allen bisher untersuchten Tumorpatientengruppen $[15,16]$ eine hochsignifikante $(p<0,000001)$ Korrelation zwischen dem mittleren Prozentsatz der repp86-positiven Tumorzellen und der Überlebenschancen bis etwa 20 Jahre nach der Diagnose und Therapie, die beispielsweise im Falle der Patientinnen mit T1 \& 2, N0, M0 Mammakarzinomen in einer «Lumpectomy» und lokalen Radiatio besteht (Abb. 2).

In Abhängigkeit von der prognostischen Treffergenauigkeit des Markers lassen sich für die Kalibrierung einer invasiven Therapie wichtige Entscheidungen treffen, und vor allem lässt sich eine «Überbehandlung» mit Zytostatika vermeiden. Obwohl die moderne Bioinformatik bei solchen Studien sogenannte zentrale Tendenzen mit entsprechenden Vertrauensbereichen herausarbeitet, behalten die Aussagen auch für das Individuum ihre Gültigkeit So stimmen die Überlebenschancen von Patientinnen mit T1 \& T2, N0, M0 Mammakarzinom und einer repp86-Proliferationsrate von $<10 \%$ der Tumorzellen laut einer im Journal of the National Cancer Institute publizierten Studie mit der gleichaltriger gesunder Frauen überein [16].

In derselben Studie starben fast alle Patientinnen, deren Tumorzellen zu $>10 \%$ das Protein repp86 enthielten. Solche Informationen leisten wertvolle Dienste, indem sie uns helfen, weniger aggressive Tumoren, die mit der konventionellen 
Tab. 3. Proliferationsmarker als Prognosefaktoren bei unterschiedlichen Tumorentitäten

\begin{tabular}{|c|c|c|c|c|c|c|c|c|}
\hline \multirow[t]{2}{*}{ Tumor-Entität } & \multirow[t]{2}{*}{ Fallzahl } & \multirow{2}{*}{$\begin{array}{l}\text { Beobachtungs- } \\
\text { Zeitraum, } \\
\text { Monate }\end{array}$} & \multirow[t]{2}{*}{ Marker } & \multicolumn{3}{|c|}{ Signifikanz, p } & \multirow[t]{2}{*}{ UPF } & \multirow[t]{2}{*}{ Referenz } \\
\hline & & & & GÜ & BÜ & RÜ & & \\
\hline Mammakarzinom & 142 & 144 & $\mathrm{Ki}-\mathrm{S} 1$ & $<0,001$ & n.u. & $<0,001$ & + & {$[6]$} \\
\hline Mammakarzinom & 83 & 60 & $\mathrm{Ki}-\mathrm{S} 1$ & n.u. & n.u. & $<0,0001$ & + & [7] \\
\hline \multirow[t]{2}{*}{ Mammakarzinom } & 356 & 208 & Ki-S5 & 0,00005 & 0,00001 & $<0,00001$ & + & {$[21]$} \\
\hline & & & $\mathrm{Ki}-\mathrm{S} 4$ & $<0,00001$ & $<0,00001$ & 0,00004 & + & \\
\hline \multirow[t]{2}{*}{ Mammakarzinom } & 863 & 200 & $\mathrm{Ki}-\mathrm{S} 4$ & $<0,0001$ & n.u. & $<0,0001$ & + & {$[22]$} \\
\hline & & & Ki-S11 & $<0,0001$ & n.u. & $<0,0001$ & + & \\
\hline Mammakarzinom & 371 & 208 & $\mathrm{Ki}-\mathrm{S} 2$ & $<0,0001$ & $<0,0001$ & $<0,0001$ & + & {$[16]$} \\
\hline Weichteilsarkome & 132 & 170 & Ki-S11 & 0,0005 & n.u. & 0,0004 & + & {$[11]$} \\
\hline GIST & 52 & 144 & Ki-S5 & n.u. & $<0,00001$ & 0,0012 & + & {$[12]$} \\
\hline Neuroblastom & 101 & 136 & $\mathrm{Ki}-\mathrm{S} 5$ & $<0,00001$ & n.u. & $<0,00001$ & + & {$[8]$} \\
\hline Neuroblastom & 161 & 191 & $\mathrm{Ki}-\mathrm{S} 5$ & n.u. & n.u. & $<0,00001$ & + & [9] \\
\hline Hypopharynx-Karzinom & 131 & 72 & Ki-S11 & 0,01 & n.u. & n.u. & n.u. & {$[23]$} \\
\hline Melanom & 167 & 87 & Ki-S5 & n.u. & n.u. & $<0,0001$ & + & {$[24]$} \\
\hline Endometrium-Karzinom & 164 & 151 & $\mathrm{Ki}-\mathrm{S} 4$ & n.u. & 0,03 & n.u. & + & {$[14]$} \\
\hline Endometrium-Karzinom & 164 & 151 & $\mathrm{Ki}-\mathrm{S} 2$ & n.u. & 0,007 & n.u. & + & {$[17]$} \\
\hline
\end{tabular}

GÜ = Gesamtüberleben; BÜ = bereinigtes Überleben (disease-specific survival); RÜ = rezidivfreies Überleben; UPF = unabhängiger Prognosefaktor (multivariant); n.u. = nicht untersucht; GIST = gastrointestinaler Stromatumor.

Morphologie nicht abgrenzbar sind, vor Therapiebeginn auszumachen und Patientinnen vor Überbehandlung zu schützen. Für jeden Tumor können solche Zusatzinformationen bereits bei der Schnellschnittdiagnose etwa 15 min nach der chirurgischen Probenentnahme unter geeigneten Bedingungen verfügbar gemacht werden. Ähnliche Ergebnisse sind für Korpuskarzinome des Uterus ermittelt worden [17].

Dem Pathologen werden zunehmend komplexere Informationen abverlangt. Häufig muss die Diagnose über eine solide morphologische Analyse hinaus auch immunphänotypische, zytogenetische und molekulargenetische Erkenntnisse umfassen, um den Erfordernissen der modernen klinischen Onkologie zu genügen. Am augenfälligsten wird dies an den hämatopathologischen Neoplasien. Hier sind zusätzlich immunhistochemische Informationen unumgänglich, um zu einer verlässlichen Diagnose zu gelangen.

Ein Malignom lässt sich als eine Neoplasie des hämatopoetischen Systems klassifizieren wenn es neben den üblichen histomorphologischen und zytologischen Kriterien einen Immunphänotyp $\mathrm{CD}^{+} 5^{+}$, Zytokeratin ${ }^{-}$oder S100- aufweist. Dabei darf nicht außer Acht bleiben, dass bei manchen hämatopoetischen Neoplasien CD45 fehlen kann (Tab. 2). Hierzu gehören manche stammzellnahe akute myeloische und lymphoblastische Leukämien und Lymphome. Aber auch PlasmazellMyelome, solitäre Plasmozytome, echte maligne Histiozytosen, anaplastische hochmaligne Lymphome (ALK1-Lymphome, Ki-1-Lymphome) können das CD45 vermissen lassen.

Die myelomonozytären Neoplasien, wie die entsprechenden akuten und chronischen Leukämien, die sogenannten Myelosarkome oder die monozytären Sarkome lassen sich nicht nur auf dem Boden morphologischer Kriterien, sondern auch immunphänotypisch durch $\mathrm{CD}^{+} 5^{+}$, Ki-My1,2 und/oder $3^{+}$, KiM1 $\mathrm{p}^{+}$identifizieren. Reifere Formen exprimieren die Myeloperoxidase und/oder die monozyten-spezifische Naphthol-ASAcetatesterase, die sowohl enzymzytochemisch und durch Isoenzymanalyse als auch immunhistochemisch mit entsprechenden monoklonalen Antikörpern erkannt werden können. Monozytäre Differenzierung kann auch durch den Nachweis eines monozytenspezifischen Methylierungsmusters der Promotorregion des fms-Onkogens bewiesen werden. Das Onkogen $c$-fms kodiert für das Onkoprotein fms, den Rezeptor für den Makrophagen-Wachstumsfaktor M-CSF.

Hämatopathologische Diagnosen bedürfen jedoch oft weiterer Verfeinerung, entweder durch erweiterte Immunhistochemie, Zytogenetik oder Molekulargenetik. So kann die anhand morphologischer und immunhistochemischer Befunde vermutete Diagnose einer Mycosis fungoides (kutanes T-Zell-Lymphom) im Frühstadium meist nur durch den molekulargenetischen Nachweis eines klonalen Rearrangements des T-ZellRezeptors gesichert werden. Auch können bestimmte Gentranslokationen, -amplifikationen oder -fusionen, wie z.B. die Translokation $\mathrm{t}(11 ; 14)$ beim Mantelzell-Lymphom oder das Fusionsgen NPM-ALK beim großzelligen anaplastischen Lymphom (Ki1-Lymphom) diagnostisch wegweisend sein und darüber hinaus auch Aufschlüsse über die Prognose geben. In der Differentialdiagnostik von Weichteilsarkomen werden diese Techniken ebenfalls mit Erfolg eingesetzt.

Ein ganz neuer Aspekt in der molekularen Tumordiagnostik ist die Bestimmung der Telomerase und die Quantifizierung ihrer enzymatischen Aktivität an bioptischem Untersuchungs- 
gut. Dieses während der Embryonalperiode aktive Enzym wird in ausgereiften somatischen Zellen des erwachsenen Organismus abgeschaltet, was eine präzise Begrenzung des Zellwachstums zur Folge hat. Bei manchen Tumoren kann jedoch das Gen in unterschiedlichem Ausmaß reaktiviert werden. Das bedeutet, dass Tumoren, die das Gen nicht - oder nur in geringem Maße - exprimieren, nur ein begrenztes Wachstumspotential haben und letztendlich in eine Regression eintreten. Umgekehrt ist von Tumoren mit hoher Telomeraseaktivität eine ungehemmte Ausbreitung zu erwarten. Diese zunächst hypothetischen Betrachtungen haben inzwischen durch etliche In-vivo-Untersuchungen Bestätigung gefunden. Bei melanozytären Tumoren erlaubt die quantitative Bestimmung der Telomerase mit hoher Trennschärfe eine Unterscheidung benigner und maligner Veränderungen inklusive ihrer Vorstufen und gewährleistet zuverlässige Voraussagen über den Krankeitsverlauf [18]. Beim Endometriumkarzinom erwies sich die Telomeraseaktivität als weitaus potentester unabhängiger Prognosefaktor [19] im Vergleich mit etablierten Kriterien und sogar der Proliferationsaktivität [17]. Beim Neuroblastom, dem häufigsten soliden Tumor des Kindesalters, ist die Telomeraseaktivität wahrscheinlich mehr als jeder andere histologische, zytologische, immunhistochemische oder molekulargenetische Parameter ausschlaggebend für den Krank- heitsverlauf [20]. Angesichts der Bemühungen zur Entwicklung von Pharmaka zur Inhibition der Telomerase dürfte solchen Untersuchungen in naher Zukunft immer größere Bedeutung zukommen.

\section{Schlussfolgerung}

Die Pathologie steht somit im Brennpunkt einer explosionsartigen Evolution diagnostischer und prognostischer Technologien, deren Anwendung in zunehmendem Maß der ärztlichen Versorgung des Patienten zugute kommt. Die Aufgabe der Pathologie ist dabei nicht darauf beschränkt, dem neuesten Standard diagnostischer Methoden Genüge zu tun, vielmehr spielt sie eine zentrale Rolle in der Entwicklung neuer Verfahren und in der Validierung aktueller Forschungsergebnisse. Wenngleich sich die Arbeitsstrecke von der ersten morphologischen Begutachtung bis zur endgültigen Diagnose unter Berücksichtigung aller mit unterschiedlichen Methoden erhobener Daten immer mehr verlängert, ist das Ergebnis zweifelsfrei den Aufwand wert: Was heute noch eine Hoffnung ist, kann bereits morgen klinische Realität werden - eine auf Grund optimaler Diagnostik individuell auf den Patienten abgestimmte minimal invasive und maximal effiziente Therapie.

\section{Literatur}

1 Baselga J: Clinical trials of Herceptin (trastuzumab). Eur J Cancer 2001;37(suppl 1):18-24.

2 Rudolph P, Alm P, Olsson H, Heidebrecht HJ, Ferno M, Baldetorp B, Parwaresch R: Concurrent overexpression of $\mathrm{p} 53$ and c-erbB-2 correlates with accelerated cycling and concomitant poor prognosis in node-negative breast cancer. Hum Pathol 2001;32:311-319.

3 Rudolph P, Kellner U, Schmidt D, Kirchner V, Talerman A, Harms D, Parwaresch R: Ki-A10, a novel spermatogenesis-associated antigen retained in a subset of germ cell-derived tumors. Am J Pathol 1999;154:795-803.

4 Rudolph P, Schubert B, Wacker HH, Parwaresch R, Schubert C: Immunophenotyping of dermal spindle-cell tumors: Diagnostic value of monocyte marker Ki-M1p and histogenetic considerations. Am J Surg Pathol 1997;21:791-800.

5 Rudolph P, Lappe T, Schubert C, Schmidt D, Parwaresch RM, Christophers E: Diagnostic assessment of two novel proliferation-specific antigens in benign and malignant melanocytic lesions. Am J Pathol 1995;147:1615-1625.

6 Sampson SA, Kreipe H, Gillett CE, Smith P, Chaudary MA, Khan A, Wicks K, Parwaresch R, Barnes DM: Ki-S1-a novel monoclonal antibody which recognizes proliferating cells: Evaluation of its relationship to prognosis in mammary carcinoma. J Pathol 1992;168:179-185.

7 Kreipe H, Alm P, Olsson H, Hauberg M, Fischer L, Parwaresch R: Prognostic significance of a formalin resistant nuclear proliferation antigen in mammary carcinomas as determined by the monoclonal antibody Ki-S1. Am J Pathol 1993;142:651-657.
8 Rudolph P, Lappe T, Hero B, Berthold F, Parwaresch R, Harms D, Schmidt D: Prognostic significance of the proliferative activity in neuroblastoma. Am J Pathol 1997;150:133-145.

9 Krams M, Hero B, Berthold F, Parwaresch R, Harms D, Rudolph P: Proliferation marker Ki-S5 discriminates between favourable and adverse prognosis in advanced stages of neuroblastoma with and without MYCN amplification. Cancer, in press 2002.

10 Papadopoulos I, Rudolph P, Weichert-Jacobsen K: Value of p53 expression, cellular proliferation and DNA content as prognostic indicators in renal cell carcinoma. Eur Urol 1997;32:110-117.

11 Rudolph P, Kellner U, Chassevent A, Collin F, Bonichon F, Parwaresch R, Coindre JM: Prognostic relevance of a novel proliferation marker, Ki-S11, for soft tissue sarcoma. A multivariate study. Am J Pathol 1997;150:1997-2007.

12 Rudolph P, Gloeckner K, Parwaresch R, Harms D, Schmidt D: Immunophenotype, proliferation, DNA ploidy and biological behavior of gastrointestinal stromal tumors. A multivariate clinicopathologic study. Hum Pathol1998;29:791-800.

13 Rudolph P, Schubert C, Schubert B, Parwaresch R Proliferation marker Ki-S5 as a diagnostic tool in melanocytic lesions. J Am Acad Dermatol 1997;37: 169-178.

14 Bonatz G, Lüttges J, Hamann S, Mettler L, Jonat W, Parwaresch R: Immunohistochemical assessment of p170 provides prognostic information in endometrial carcinoma. Histopathology 1999;34: $43-50$.
15 Gerdes J, Dallenbach F, Lennert K, Lemke H, Stein H: Growth fractions in malignant nonHodgkin's lymphomas (NHL) as determined in situ with the monclonal antibody Ki-67. Hematol Oncol 1984;2:365-371.

16 Rudolph P, Alm P, Heidebrecht HJ, Bolte H, Ratjen V, Baldetorp B, Ferno M, Olsson H, Parwaresch $\mathrm{R}$ : Immunologic proliferation marker Ki-S2 as prognostic indicator for lymph node-negative breast cancer. J Natl Cancer Inst 1999;91:271-278.

17 Bonatz G, Luttges J, Hedderich J, Inform D, Jonat W, Rudolph P, Parwaresch R: Prognostic significance of a novel proliferation marker, anti- repp 86, for endometrial carcinoma: A multivariate study. Hum Pathol 1999;30:949-956.

18 Rudolph P, Schubert C, Tamm S, Heidorn K, Hauschild A, Michalska I, Majewski S, Krupp G, Jablonska S, Parwaresch R: Telomerase activity in melanocytic lesions: A potential marker of tumor biology. Am J Pathol 2000;156:1425-1432.

19 Bonatz G, Frahm SO, Klapper W, Helfenstein A, Heidorn K, Jonat W, Krupp G, Parwaresch R, Rudolph P: High telomerase activity is associated with cell cycle deregulation and rapid progression in endometrioid adenocarcinoma of the uterus. Hum Pathol 2001;32:605-614.

20 Krams M, Claviez A, Heidorn K, Krupp G, Parwaresch R, Harms D, Rudolph P: Regulation of telomerase activity by alternate splicing of human telomerase reverse transcriptase mRNA in a subset of neuroblastomas. Am J Pathol 2001;159:19251932. 
21 Rudolph P, Olsson H, Bonatz G, Ratjen V, Bolte H, Baldetorp B, Fernö M, Parwaresch R, Alm P: Correlation between $\mathrm{p} 53$, c-erbB-2, and topoisomerase II alpha expression, DNA ploidy hormonal receptor status and proliferation in 356 node-negative breast carcinomas: Prognostic implications. J Pathol 1999;187:207-216.

22 Rudolph P, MacGrogan G, Bonichon F, Frahm SO, deMascarel L, Trojani M, Durand M, Avril A, Coindre JM, Parwaresch R: Prognostic significance of $\mathrm{Ki}-67$ and topoisomerase II alpha expression in infiltrating ductal breast cancer. A multivariate analysis of 863 cases. Breast Cancer Res Treat 1999; 55:61-71.
23 Kuropkat C, Rudolph P, Frahm SO, Parwaresch R, Werner JA: Proliferation marker Ki-S11 a prognostic indicator for squamous cell carcinoma of the hypopharynx. Virchows Arch 1999;435:590-595.

24 Frahm SO, Schubert C, Parwaresch R, Rudolph P: High proliferative activity may predict early metastasis of thin melanomas. Hum Pathol 2001;32:13761381 .
25 Parwaresch R, Rudolph P: The cell cycle-theory and application to cancer. Onkologie 1996;19:464472.

26 Kellner U, Rudolph P, Parwaresch R: Human DNA-topoisomerases - diagnostic and therapeutic implications for cancer. Onkologie 2000;23:424430. 